\title{
Oxygen management among infants in neonatal units in sub-Saharan Africa: a cross-sectional survey
}

\author{
Scott K. Herrod ${ }^{1} \cdot$ Alex Stevenson $\mathbb{D}^{2} \cdot$ Yvonne E. Vaucher $^{3} \cdot$ Scott R. Lambert ${ }^{4}$ - Sherwin J. Isenberg ${ }^{5}$. \\ Vivien L. Yap $\mathbb{1}^{6} \cdot$ V. Chinyere Ezeaka ${ }^{7} \cdot$ Waldemar A. Carlo $\mathbb{1}^{8}$
}

Received: 20 November 2020 / Revised: 25 February 2021 / Accepted: 5 March 2021 / Published online: 26 March 2021

(c) The Author(s), under exclusive licence to Springer Nature America, Inc. 2021

\begin{abstract}
Objectives To provide more comprehensive data on the management of oxygen supplementation in neonates in sub-Saharan Africa.

Study design An online survey on the management of oxygen supplementation for infants in neonatal units was sent to 278 healthcare personnel in sub-Saharan Africa.

Results One hundred and nine responses from 82 neonatal care units in 54\% (26/48) sub-Saharan African countries were received. All units had the capacity to provide oxygen supplementation. However, only 50\% (38/76) had access to blend oxygen with medical air and 1\% (1/75) had the capacity to blend oxygen/air for every infant. Although 96\% (72/75) of units could monitor oxygen saturation, monitoring was mostly intermittent and only 32\% (24/75) were able to monitor oxygen saturation in every infant receiving oxygen supplementation.

Conclusions Findings indicate that oxygen supplementation is inadequately managed in neonatal units in sub-Saharan Africa, which may put infants at risk of developing severe ROP.
\end{abstract}

\section{Introduction}

Supplemental oxygen is necessary to treat respiratory distress syndrome, a frequent life-threatening disease in infants born prematurely; however, careful oxygen management is important to reduce the incidence of ROP [1]. There has been an epidemic of blindness due to retinopathy of

\footnotetext{
Supplementary information The online version contains supplementary material available at https://doi.org/10.1038/s41372021-01040-7.

Waldemar A. Carlo

wcarlo@peds.uab.edu

Brigham Young University, Provo, UT, USA

2 University of Zimbabwe, Harare, Zimbabwe

3 University of California San Diego, San Diego, CA, USA

4 Stanford University School of Medicine, Palo Alto, CA, USA

5 UCLA School of Medicine, Los Angeles, CA, USA

6 Weill Cornell Medicine, New York, NY, USA

7 University of Lagos College of Medicine, Lagos, Nigeria

8 University of Alabama at Birmingham, Birmingham, AL, USA
}

prematurity (ROP) in middle-income countries that started within the last 25 years [2]. In some countries, ROP contributed to more than $50 \%$ of childhood blindness and blinded thousands of children [3]. The administration of unblended (100\%) supplemental oxygen contributes to more severe ROP in preterm infants in middle-income countries [4]. A meta-analysis of older studies has reported that the restriction of oxygen supplementation decreased the incidence of ROP [5] and a recent meta-analysis of five randomized, controlled trials in extremely preterm infants showed that oxygen saturation targets of $91-95 \%$ led to more treatment-warranted ROP but not more blindness compared to targets of 85-89\% [6]. These trials were conducted in high-income countries where timely retinal examination and treatment for ROP were routinely available. Most importantly, the lower oxygen saturation targets increased mortality and necrotizing enterocolitis [6]. In the largest randomized controlled trial of oxygen saturation targeting, higher achieved oxygen saturations were associated with increased risk for ROP [7]. Continuous monitoring of oxygen saturation in preterm infants is essential to maximize the time an infant is within an appropriate oxygen saturation target range to avoid visual impairment and blindness due to ROP while also preventing death. Lower 
patient to nurse ratios in neonatal intensive care units increase the amount of time a patient is within oxygen saturation targets [8].

It has been suggested that blindness from ROP increases in conjunction with the introduction and expansion of neonatal care [2]. Initiatives to reduce ROP in low- and middle-income countries by improving oxygen management have been reported [9]. Africa has been deemed the "New Frontier" for ROP due to the continual expansion of neonatal care [10]. However, little is known about how oxygen is currently managed in neonatal units throughout sub-Saharan Africa. Recent reports indicate that units in sub-Saharan Africa have high patient to nurse ratios [11, 12] likely making oxygen saturation monitoring difficult. Previously, we reported on the management of ROP in preterm infants in sub-Saharan Africa [13]. Responses from 28 neonatologists from 8 sub-Saharan Africa countries revealed that $64 \%$ did not have the capacity to reduce supplemental oxygen concentration by blending oxygen with air. Although these data are informative, the primary focus of the previous publication was on ROP management and not oxygen management. It also lacked responses from many countries within sub-Saharan Africa, including French and Portuguese speaking nations.

The primary objectives of this study were to (1) provide more comprehensive data on oxygen administration and saturation monitoring in neonates in sub-Saharan Africa and (2) identify differences in these measures in neonatal care units with and without neonatologists.

\section{Subjects and methods}

The International Pediatric Ophthalmology and Strabismus Council (IPOSC) recently established an ROP Task Force entitled "Stop Infant Blindness in Africa" (SIBA) and is comprised of more than thirty ophthalmologists from many countries around the world. SIBA's mission is to reduce preventable blindness from ROP in preterm infants in subSaharan Africa. This will be accomplished through needs assessments, training, providing equipment, and collaboration with physicians in sub-Saharan Africa. A Neonatology Advisory Committee was also formed to advise the SIBA committee, and most of the neonatologists on this committee have extensive experience working with neonatal nurseries in sub-Saharan Africa.

As part of the SIBA initiative, an online cross-sectional survey was created with the purpose of querying neonatal staff on current methods of oxygen administration including number of infants receiving oxygen supplementation, air and oxygen sources, methods of blending oxygen/air, respiratory support devices, oxygen saturation targets and alarm limits, duration of monitoring, and the role of nurses in oxygen management within neonatal care units in subSaharan Africa. The Stanford University Institutional Review Board approved the protocol as exempt.

The contact information of neonatal healthcare workers, including neonatologists, pediatricians, and neonatal nurses, was collected between April 27-July 31, 2020. The email and/or WhatsApp Messenger information of these individuals were gathered through a previously created conference attendee list, corresponding authors of papers pertaining to neonatology in sub-Saharan Africa, secondary contacts of these authors, African pediatric and neonatal societies, online resources, and personal contacts. We attempted to reach as many neonatal care units in subSaharan Africa as possible. In total, 282 individuals were surveyed.

The questionnaire was created in English in Qualtrics Survey Software (Provo, UT, USA), distributed via email and/or WhatsApp Messenger and accessed through a hyperlink. The 32 questions in the survey were developed by the SIBA committee and the Neonatal Advisory Committee members in collaboration with an African neonatologist. The questionnaire was field tested in Zimbabwe and modified prior to submission to the Institutional Review Board. The questionnaire was translated into French and Portuguese to prevent the data being biased towards English-speaking nations. The survey was anonymous in order to encourage accurate responses.

Responses were collected between July 15 and August 4, 2020. Each contact received a message introducing SIBA, the purpose of the questionnaire, the estimated length of time for completion, and an explanation that their responses would remain anonymous. In many instances this message was the initial contact with the participant. No incentives were offered to participants, but the survey results will be made available to participants following publication. To prevent duplicate responses, each participant received a single personal hyperlink that could be used to submit a response one time. Respondents were able to continue to access the survey through their personal hyperlink in order to view the questions until they submitted their response. Survey items were not randomized or based on adaptive questioning. Each page consisted of three to six items and the survey was distributed over seven pages. A Back button was provided on each page until submission of the survey in case a participant wanted to change their response. Participants were also notified of any unanswered questions before advancing to the next page. Respondents were not forced to complete every question prior to submitting the survey.

In six instances respondents reported inadvertently submitting an incomplete response and requested a new link. In order to prevent duplicate responses, the participant helped to identify their previous response (to exclude it from 
analysis) and were sent a new link. Participants received up to two reminder messages if no initial response was received. There was no timeframe used as a cut-off point when determining if certain responses needed to be excluded from analysis. Four responses were excluded from analysis due to many incomplete responses or one response being from more than one unit. Responses that included very unlikely or implausible data entry errors were excluded from the analysis because the anonymity of the survey did not allow follow-up questions for clarification. The mean was used in analysis of range of values provided for point estimates. If a respondent included a value combined with terms such as "more than," "about," "around," "or less," etc., then the words were omitted, and the number indicated was used for analysis. The variable denominators include only valid responses; thus, the denominator varies from variable to variable.

Countries were determined to be either English, French, or Portuguese speaking depending on the official language or which of the three languages was most commonly spoken. For units with more than one respondent, the responses were consolidated into one composite response in order to prevent overrepresentation. For numerical data, the mean of the responses was used in the composite response. For multiple choice and ordinal data, the majority response was used. If a tie between choices occurred, the response to the question was excluded from analysis. In "mark all that apply" questions, all answers were included in the composite response unless one choice excluded other choices. Blank responses to questions were not included in the analysis.

Microsoft Excel (Redmond, WA, USA) and R Statistical Software (Vienna, Austria) were used to analyze the data and construct tables. Shapiro-Wilk normality tests revealed that the continuous data was positively skewed and not normal. Therefore, nonparametric comparison between groups using the Wilcoxon signed rank-sum tests were used when comparing continuous data. The Median and range of continuous data were reported for center values as they were calculated with the Wilcoxon results. Chi-squared $\left(\chi^{2}\right)$ tests were used when comparing proportional data. All statistical tests were two-tailed. In total, 56 statistical tests were performed. To reduce the likelihood of Type I error, a Bonferroni Correction was calculated to reset the significance cut-off. The correction was calculated by dividing 0.05 by the total number of statistical tests (56) to reset the alpha to 0.0009 , making $p \leq 0.0009$ statistically significant. ArcGIS Pro software (Redlands, CA, USA) was used to create geospatial maps depicting the location of responses within sub-Saharan Africa.

To assess what was previously published on oxygen management in neonatal units in sub-Saharan Africa, a literature search was conducted on August 25 and September
16, 2020. Search items included a combination of terms such as "oxygen management," "oxygen," "neonatal unit," "survey" and/or "Africa." Studies published since the year 2000 were included in the review. Google Scholar, PubMed and Embase were the primary search engine used.

This report has been formatted according to the Checklist for Reporting Results of Internet E-surveys (CHERRIES) guidelines.

\section{Results}

One hundred and nine responses were received from 278 contacts to whom the survey was distributed; 43 (39\%) were neonatologists, $36(33 \%)$ general pediatricians with a specialized interest in neonatology, $15(14 \%)$ general pediatricians, $9(8 \%)$ neonatal nurses, and $6(6 \%)$ healthcare workers from other specialties. In total, we received data from personnel from 82 neonatal care units in 26 of 48 (54\%) sub-Saharan African countries (Fig. 1). Responses were generated from 15 of 23 (65\%) English-speaking and 11 of $20(55 \%)$ French-speaking nations in sub-Saharan Africa. Seventeen (21\%) units were from Nigeria-the most units in a single country. Cumulatively, an estimated 112,895 infants received care in all of these neonatal care units in 2019. Neonatal care units with at least one neonatologist had more radiant warmers, incubators, and cots compared to units without a neonatologist. There were more nurses available during the day and night in units with a neonatologist compared to units without a neonatologist (Table 1). Within units with and without a neonatologist, fewer nurses were available at night compared to during the day (8 vs 4 with neonatologist, Paired Wilcoxon, $W=561$, $p<0.0009 ; 4$ vs. 2 without neonatologist; Paired Wilcoxon, $W=378, p<0.0009$ ).

All units had the capacity to provide supplemental oxygen (Table 2). In total, 53\% (40/76) of units did not have the capacity to provide medical air; $43 \%$ (19/44) of units with a neonatologist and $66 \%(21 / 32)$ of units without a neonatologist. Only $44 \%$ (33/75) of all units had the capacity to blend oxygen/air for some infants on supplemental oxygen including 54\% (22/41) units with a neonatologist and 32\% $(11 / 34)$ of units without a neonatologist. Only a single unit surveyed $(1 / 75,1 \%)$ had the capacity to provide blended oxygen/air for every infant receiving supplemental oxygen. Both neonatal units with and without a neonatologist had a median of 0 wall oxygen blenders and the most common method of blending oxygen with air in both units with and without a neonatologist was through a blender integrated into a continuous positive airway pressure (CPAP) device or ventilator.

The most common oxygen delivery instrument for all units was low-flow nasal cannula followed by CPAP and 
Fig. 1 Neonatal care units in sub-Saharan Africa who responded to our survey. Some neonatal care units were in close proximity to each other and the individual points may not be distinguished from one another.

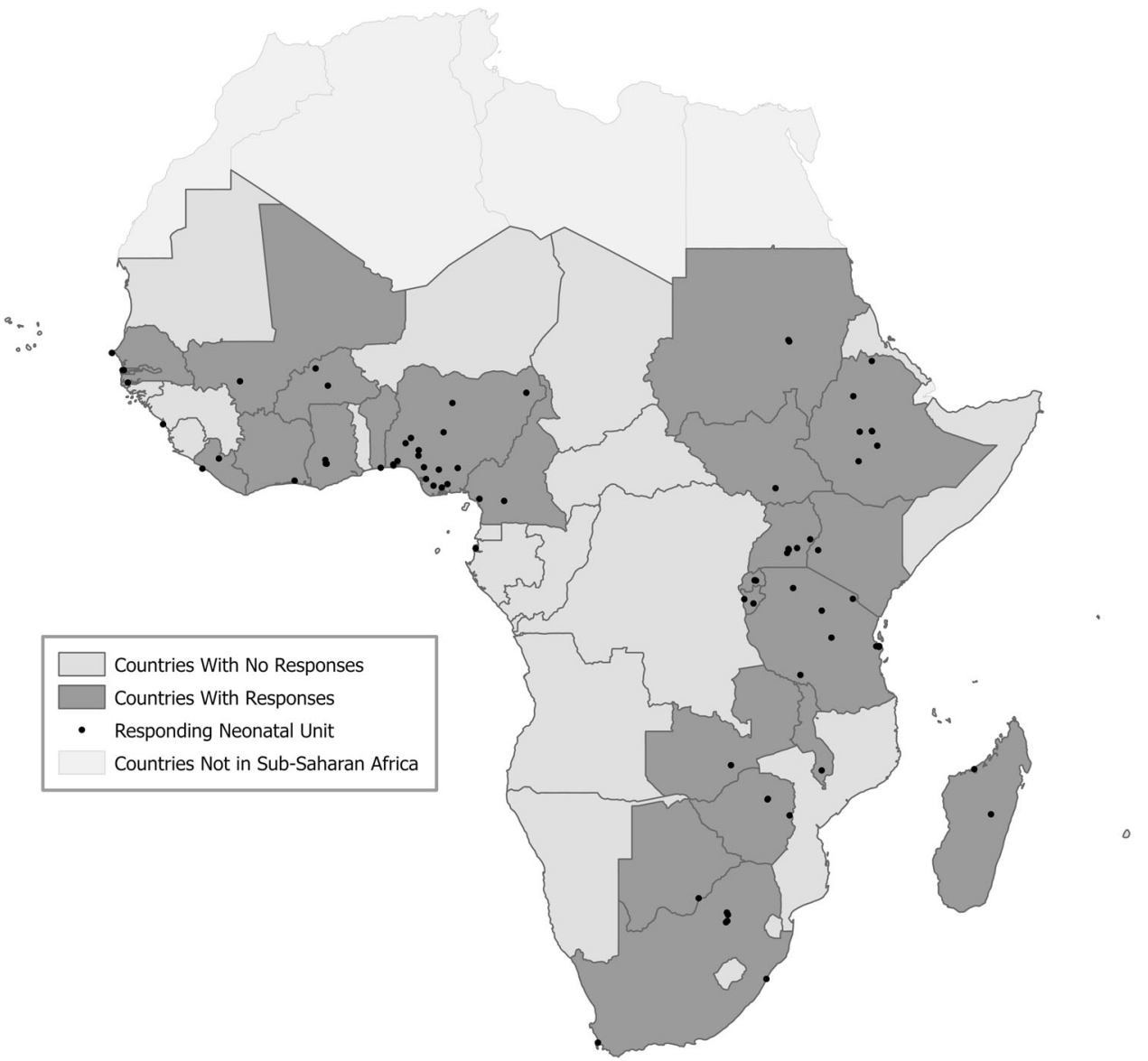

the median number of oxygen delivery equipment was highest for low-flow nasal cannula systems and CPAP devices in both units with and without a neonatologist (Table 2). Additionally, one-third of units with a neonatologist and nearly two-thirds of units without a neonatologist were not able to provide humidified oxygen/air for any infants on high flow nasal cannula, CPAP, or ventilators in their unit. Furthermore, only $10 \%$ of units with a neonatologist and $4 \%$ of units without a neonatologist were able to provide heated humidified oxygen/air for every infant.

Only 37\% (29/78) of units surveyed had an oxygen management protocol in place (Table 3). Oxygen saturation monitoring for some infants was reported in most units but only $32 \%(24 / 75)$ of units monitor every infant receiving oxygen supplementation. Most of the monitoring was reported to be intermittent. Preterm oxygen saturation targets did not differ by having a neonatologist or not. Preterm pulse oximeter alarm limits were used in 78\% (35/45) of units.

\section{Discussion}

Although all units included in this survey had the capability to provide supplemental oxygen, only $1 \%$ of the units had the capacity to blend oxygen/air for every infant receiving supplemental oxygen and $32 \%$ of units had the capacity to monitor oxygen saturation in every infant receiving supplemental oxygen. Most were only able to monitor oxygen saturation intermittently, limiting the effectiveness of oxygen saturation monitoring. Additionally, on average neither units with or without a neonatologist have adopted the recommended World Health Organization guidelines of maintaining oxygen saturation in preterm infants between $88-95 \%$ and not higher than $95 \%$ to prevent the development of ROP [14].

Limitations to this study include a low-response rate (39\%), disproportionate responses from different countries in sub-Saharan Africa, and the units in the survey may not accurately reflect the current management of oxygen supplementation within each country, all of which limit the generalizability of the results. Respondents may also have been subject to recall bias, which could have led to underor over-estimating the availability of resources and number of infants receiving care in their unit. Despite these limitations, this study includes more countries than any other study on oxygen management in neonatal care units in subSaharan Africa to the best of our knowledge. Other studies did not have as many countries represented [13], were 
Table 1 Neonatal care units with or without neonatologist.

\begin{tabular}{|c|c|c|c|c|}
\hline & & With a neonatologist & $\begin{array}{l}\text { Without a } \\
\text { neonatologist }\end{array}$ & $P$ value \\
\hline Number of units & & 47 & 35 & \\
\hline Number of countries & & 20 & 17 & \\
\hline $\begin{array}{l}\text { Admissions in } 2019, \text { median } \\
(\min , \max )\end{array}$ & & $\begin{array}{l}1322(70,8000), \\
(n=44)\end{array}$ & $\begin{array}{l}779(50,3500), \\
(n=28)\end{array}$ & $\mathrm{NS}^{\mathrm{a}, \mathrm{b}}$ \\
\hline \multirow[t]{4}{*}{ Daily census } & & & & $\mathrm{NS}^{\mathrm{a}, \mathrm{c}}$ \\
\hline & $1-20$ infants, $n / N(\%)$ & $15 / 43(35 \%)$ & $19 / 34(56 \%)$ & \\
\hline & $21-50$ infants, $n / N(\%)$ & $18 / 43(42 \%)$ & $12 / 34(35 \%)$ & \\
\hline & $\geq 51$ infants, $n / N(\%)$ & $10 / 43(23 \%)$ & $3 / 34(9 \%)$ & \\
\hline Bed capacity, median (min max) & & $40(7,185),(n=47)$ & $24(3,70),(n=34)$ & $\mathrm{NS}^{\mathrm{a}, \mathrm{b}}$ \\
\hline \multicolumn{5}{|l|}{ Bed type } \\
\hline & $\begin{array}{l}\text { Radiant warmers, median } \\
(\min , \max )\end{array}$ & $6(0,50),(n=47)$ & $3(0,10),(n=35)$ & $W=1219, p<0.0009^{b}$ \\
\hline & Incubators, median (min, max) & $10(0,125),(n=47)$ & $4(0,24),(n=34)$ & $W=1213, p<0.0009^{\mathrm{b}}$ \\
\hline & Cots, median (min, max) & $22(0,120),(n=47)$ & $10(0,68),(n=33)$ & $W=1123, p<0.0009^{\mathrm{b}}$ \\
\hline \multicolumn{5}{|l|}{ Number of nurses } \\
\hline & Day, median (min, max) & $8(1,50),(n=45)$ & $4(1,10),(n=35)$ & $W=1251, p<0.0009^{\mathrm{b}}$ \\
\hline & Night, median (min, max) & $4(1,33),(n=46)$ & $2(1,8),(n=35)$ & $W=1153, p<0.0009^{\mathrm{b}}$ \\
\hline
\end{tabular}

${ }^{a}$ Not significant, ( $p$ value $>0.0009$ ), based on Bonferroni Correction.

${ }^{\mathrm{b}} p$ value calculated with Wilcoxon signed rank-sum test.

${ }^{c} p$ value calculated with Chi-squared $\left(\chi^{2}\right)$ test.

conducted in individual countries $[15,16]$, or were not specifically targeting neonatal care units in sub-Saharan Africa [17]. Additionally, it is likely that the neonatal units of non-responding healthcare personnel were no better equipped to manage oxygen supplementation than responding units.

The inability to provide blended oxygen/air for every infant in all but one unit surveyed is of immediate concern. Infants who receive unrestricted oxygen in units without the capacity to blend oxygen/air are at increased risk of developing severe ROP [4]. This may contribute to the observations from previous studies that larger and/or more mature infants ( $\geq 1500 \mathrm{~g}$ and/or $\geq 32$ weeks gestational age) are developing ROP in sub-Saharan Africa [18-25]. A compressed oxygen source was available in every unit, but many units did not have the capacity to blend oxygen/air because of lack of compressed medical air, precluding the use of traditional blenders. Innovative blenders of oxygen/ air can be effective without the need of compressed medical air [26, 27], but some are limited due to incompatibility with nasal cannula [26] or are not yet on the market [27]. Advocacy for compressed air and oxygen sources, or instruments that can blend oxygen/air without a compressed air source, are crucial in some units in sub-Saharan Africa.

Lack of blenders and oxygen saturation monitors and high patient to nurse ratios may result in increased risk of death, necrotizing enterocolitis, and severe ROP [6].
Ophthalmologists in Africa only have limited access to laser photocoagulation [13], the current standard of care for treatment of severe ROP. Even if laser treatment was readily available in every neonatal unit, many units in sub-Saharan Africa report a high number of infants lost to follow-up $[28,29]$, which would reduce its effectiveness. Anti-VEGF medication is the most common treatment modality for ROP in sub-Saharan Africa [13], but the recurrence rate is high [30]. This suggests that infants may still become blind despite receiving treatment due to the recurrence of ROP following discharge. The limited ability to treat ROP and high number of patients lost to follow-up suggests that measures to prevent the severe form of the disease will need to be taken to curb an epidemic of blindness from ROP in Africa. This can be done through comprehensive efforts to improve the capacity to blend oxygen/air and to adequately monitor targeted oxygen saturations in infants in subSaharan Africa.

In conclusion, this study reports current oxygen management in a large number of neonatal care facilities in subSaharan Africa. Despite the availability of oxygen supplementation in all units surveyed, only one unit had the capacity to provide blended oxygen/air for every infant. This lack of blending can increase the risk of severe ROP [4]. Additionally, the limited availability and consistency of oxygen saturation monitoring may also put preterm infants in these neonatal care units at greater risk for developing 
Table 2 Respiratory support in neonatal care units with and without neonatologist.

\begin{tabular}{|c|c|c|c|c|}
\hline & & With a neonatologist & Without a neonatologist & $p$ value \\
\hline \multirow[t]{3}{*}{ Infants on oxygen per day } & & & & $\mathrm{NS}^{\mathrm{a}, \mathrm{b}}$ \\
\hline & $1-15, n / N(\%)$ & $28 / 43(65 \%)$ & $27 / 34(79 \%)$ & \\
\hline & $\geq 16, n / N(\%)$ & $15 / 43(35 \%)$ & $7 / 34(21 \%)$ & \\
\hline \multicolumn{5}{|l|}{ Oxygen source } \\
\hline & In-wall oxygen lines, $n / N(\%)$ & $34 / 47(72 \%)$ & $14 / 35(40 \%)$ & $\mathrm{NS}^{\mathrm{a}, \mathrm{b}}$ \\
\hline & Oxygen cylinders, $n / N(\%)$ & $29 / 47(62 \%)$ & $25 / 35(71 \%)$ & $\mathrm{NS}^{\mathrm{a}, \mathrm{b}}$ \\
\hline & Oxygen concentrator, $n / N(\%)$ & $29 / 47(62 \%)$ & $25 / 35(71 \%)$ & $\mathrm{NS}^{\mathrm{a}, \mathrm{b}}$ \\
\hline & Oxygen unavailable, $n / N(\%)$ & $0 / 47(0 \%)$ & $0 / 35(0 \%)$ & $\mathrm{NA}^{\mathrm{c}}$ \\
\hline \multicolumn{5}{|l|}{ Air source } \\
\hline & In-wall air lines, $n / N(\%)$ & $20 / 44(45 \%)$ & $7 / 32(22 \%)$ & $\mathrm{NS}^{\mathrm{a}, \mathrm{b}}$ \\
\hline & Air compressor, $n / N(\%)$ & $8 / 44(18 \%)$ & $5 / 32(16 \%)$ & $\mathrm{NS}^{\mathrm{a}, \mathrm{b}}$ \\
\hline & Medical air unavailable, $n / N(\%)$ & $19 / 44(43 \%)$ & $21 / 32(66 \%)$ & $\mathrm{NS}^{\mathrm{a}, \mathrm{b}}$ \\
\hline \multicolumn{5}{|l|}{ Air/oxygen blending method } \\
\hline & Wall blender, $n / N(\%)$ & $9 / 44(20 \%)$ & $3 / 32(9 \%)$ & $\mathrm{NS}^{\mathrm{a}, \mathrm{b}}$ \\
\hline & $\begin{array}{l}\text { Integrated into } \mathrm{CPAP}^{\mathrm{d}} / \text { Ventilator, } \\
n / N(\%)\end{array}$ & $24 / 44(55 \%)$ & $8 / 32(25 \%)$ & $\mathrm{NS}^{\mathrm{a}, \mathrm{b}}$ \\
\hline & Venturi, $n / N(\%)$ & $1 / 44(2 \%)$ & $0 / 32(0 \%)$ & $\mathrm{NS}^{\mathrm{a}, \mathrm{b}}$ \\
\hline & Unable to blend, $n / N(\%)$ & $17 / 44(39 \%)$ & $21 / 32(66 \%)$ & $\mathrm{NS}^{\mathrm{a}, \mathrm{b}}$ \\
\hline Number wall blenders per unit, median (min, max) & & $0(0,40),(n=41)$ & $0(0,16),(n=32)$ & $\mathrm{NS}^{\mathrm{a}, \mathrm{e}}$ \\
\hline \multicolumn{5}{|c|}{ Percentage infants on oxygen who receive blended air/oxygen } \\
\hline & $0 \%, n / N(\%)$ & $19 / 41(46 \%)$ & $23 / 34(68 \%)$ & $\mathrm{NS}^{\mathrm{a}, \mathrm{b}}$ \\
\hline & $100 \%, n / N(\%)$ & $1 / 41(2 \%)$ & $0 / 34(0 \%)$ & $\mathrm{NS}^{\mathrm{a}, \mathrm{b}}$ \\
\hline \multicolumn{5}{|l|}{ Oxygen administration method } \\
\hline & Headbox, $n / N(\%)$ & $11 / 47(23 \%)$ & $3 / 35(9 \%)$ & $\mathrm{NS}^{\mathrm{a}, \mathrm{b}}$ \\
\hline & $\mathrm{LF}^{\mathrm{f}}$ nasal cannula, $n / N(\%)$ & $43 / 47(91 \%)$ & $32 / 35(91 \%)$ & $\mathrm{NS}^{\mathrm{a}, \mathrm{b}}$ \\
\hline & $\mathrm{HF}^{\mathrm{g}}$ nasal cannula, $n / N(\%)$ & $18 / 47(38 \%)$ & $14 / 35(40 \%)$ & $\mathrm{NS}^{\mathrm{a}, \mathrm{b}}$ \\
\hline & $\mathrm{CPAP}^{\mathrm{d}}, n / N(\%)$ & $41 / 47(87 \%)$ & $21 / 35(60 \%)$ & $\mathrm{NS}^{\mathrm{a}, \mathrm{b}}$ \\
\hline & Ventilator, $n / N(\%)$ & $19 / 47(40 \%)$ & $3 / 35(9 \%)$ & $\mathrm{NS}^{\mathrm{a}, \mathrm{b}}$ \\
\hline & Incubator oxygen, $n / N(\%)$ & $1 / 47(2 \%)$ & $0 / 35(0 \%)$ & $\mathrm{NS}^{\mathrm{a}, \mathrm{b}}$ \\
\hline \multicolumn{5}{|l|}{ Equipment available } \\
\hline & Headbox, median (min, max) & $0(0,20),(n=26)$ & $0(0,16),(n=16)$ & $\mathrm{NS}^{\mathrm{a}, \mathrm{e}}$ \\
\hline & $\mathrm{LF}^{\mathrm{f}}$ nasal cannula, median (min, $\max$ ) & $15(2,100),(n=36)$ & $5(0,50),(n=29)$ & $\mathrm{NS}^{\mathrm{a}, \mathrm{e}}$ \\
\hline & $\mathrm{HF}^{\mathrm{g}}$ nasal cannula, median (min, $\left.\max \right)$ & $3(0,15),(n=26)$ & $1(0,30),(n=20)$ & $\mathrm{NS}^{\mathrm{a}, \mathrm{e}}$ \\
\hline & RAM cannula, median (min, $\max )$ & $0(0,10),(n=20)$ & $0(0,16),(n=16)$ & $\mathrm{NS}^{\mathrm{a}, \mathrm{e}}$ \\
\hline & Vapotherm, median (min, max) & $0(0,5),(n=19)$ & $0(0,2),(n=12)$ & $\mathrm{NS}^{\mathrm{a}, \mathrm{e}}$ \\
\hline & $\mathrm{CPAP}^{\mathrm{d}}$, median $(\min , \max )$ & $4(0,50),(n=39)$ & $2(0,10),(n=25)$ & $\mathrm{NS}^{\mathrm{a}, \mathrm{e}}$ \\
\hline & Ventilator, median (min, max) & $2(0,31),(n=33)$ & $0(0,4),(n=16)$ & $\mathrm{NS}^{\mathrm{a}, \mathrm{e}}$ \\
\hline \multirow[t]{2}{*}{ Humidification system } & & & & $\mathrm{NS}^{\mathrm{a}, \mathrm{b}}$ \\
\hline & No, $n / N(\%)$ & $16 / 41(39 \%)$ & $15 / 23(65 \%)$ & \\
\hline \multicolumn{5}{|l|}{ Percentage infants receiving humidified air/oxygen } \\
\hline & $0 \%, n / N(\%)$ & $13 / 39(33 \%)$ & $14 / 23(61 \%)$ & $\mathrm{NS}^{\mathrm{a}, \mathrm{b}}$ \\
\hline & $100 \%, n / N(\%)$ & 4/39 (10\%) & $1 / 23(4 \%)$ & $\mathrm{NS}^{\mathrm{a}, \mathrm{b}}$ \\
\hline
\end{tabular}

${ }^{a}$ Not significant, ( $p$ value $>0.0009$ ), based on Bonferroni Correction.

${ }^{\mathrm{b}} p$ value calculated with Chi-squared $\left(\chi^{2}\right)$ test.

${ }^{\mathrm{c}}$ Not applicable.

${ }^{\mathrm{d}}$ Continuous positive airway pressure.

${ }^{\mathrm{e}} p$ value calculated with Wilcoxon signed rank-sum test.

${ }^{\mathrm{f}}$ Low-flow.

${ }^{\mathrm{g}}$ High-flow.

treatment-warranted ROP. Consistent and continuous oxygen saturation monitoring is necessary to reduce the risk of severe ROP. The results of this study indicate that neonatal units in countries beyond the eight with previous published reports of blindness from ROP may be placing infants at increased risk of developing severe ROP. Collaboration with policymakers, hospital administrators, alongside providers of neonatal care (neonatologists, pediatricians, and 
Table 3 Oxygen saturation monitoring in neonatal care units with and without neonatologist.

\begin{tabular}{|c|c|c|c|c|}
\hline & & With a neonatologist & Without a neonatologist & $p$ value \\
\hline \multirow[t]{2}{*}{ Oxygen management protocol } & & & & $\mathrm{NS}^{\mathrm{a}, \mathrm{b}}$ \\
\hline & No, $n / N(\%)$ & $25 / 44(57 \%)$ & $24 / 34(71 \%)$ & \\
\hline $\begin{array}{l}\text { Number of pulse oximeters, median } \\
\text { (min, max) }\end{array}$ & & $4(0,72),(n=46)$ & $3(0,16),(n=34)$ & $\mathrm{NS}^{\mathrm{a}, \mathrm{c}}$ \\
\hline \multicolumn{5}{|l|}{ Saturation levels monitored } \\
\hline & $0 \%, n / N(\%)$ & $1 / 41(2 \%)$ & $2 / 34(6 \%)$ & $\mathrm{NS}^{\mathrm{a}, \mathrm{b}}$ \\
\hline & $100 \%, n / N(\%)$ & $12 / 41(29 \%)$ & $12 / 34(35 \%)$ & $\mathrm{NS}^{\mathrm{a}, \mathrm{b}}$ \\
\hline \multirow[t]{3}{*}{ Preterm infant monitoring } & & & & $\mathrm{NS}^{\mathrm{a}, \mathrm{b}}$ \\
\hline & Continuous, $n / N(\%)$ & $11 / 45(24 \%)$ & $10 / 34(29 \%)$ & \\
\hline & Intermittent, $n / N(\%)$ & $34 / 45(76 \%)$ & $24 / 34(71 \%)$ & \\
\hline \multirow[t]{3}{*}{ Preterm infant alarm limits } & & & & $\mathrm{NS}^{\mathrm{a}, \mathrm{b}}$ \\
\hline & Yes, $n / N(\%)$ & $21 / 26(81 \%)$ & $14 / 19(74 \%)$ & \\
\hline & No, $n / N(\%)$ & $5 / 26(19 \%)$ & $5 / 19(26 \%)$ & \\
\hline \multicolumn{5}{|l|}{ Preterm saturation targets } \\
\hline & $\begin{array}{l}\text { Lower, median } \\
\text { (min, max) }\end{array}$ & $90(70,96),(n=36)$ & $91(70,97),(n=22)$ & $\mathrm{NS}^{\mathrm{a}, \mathrm{c}}$ \\
\hline & $\begin{array}{l}\text { Upper, median } \\
\text { (min, max) }\end{array}$ & $95(80,100),(n=36)$ & $95(85,100),(n=22)$ & $\mathrm{NS}^{\mathrm{a}, \mathrm{c}}$ \\
\hline \multicolumn{5}{|l|}{ Preterm alarm limits } \\
\hline & $\begin{array}{l}\text { Lower, median } \\
\text { (min, max) }\end{array}$ & $88(79,91),(n=21)$ & $89(79,90),(n=14)$ & $\mathrm{NS}^{\mathrm{a}, \mathrm{c}}$ \\
\hline & $\begin{array}{l}\text { Upper, median } \\
(\min , \max )\end{array}$ & $96(92,100),(n=21)$ & $100(95,100),(n=14)$ & $\mathrm{NS}^{\mathrm{a}, \mathrm{c}}$ \\
\hline \multirow[t]{3}{*}{ Term infant monitoring } & & & & $\mathrm{NS}^{\mathrm{a}, \mathrm{b}}$ \\
\hline & Continuous, $n / N(\%)$ & $5 / 46(11 \%)$ & $6 / 33(18 \%)$ & \\
\hline & Intermittent, $n / N(\%)$ & $41 / 46(89 \%)$ & $27 / 33(82 \%)$ & \\
\hline \multirow[t]{3}{*}{ Term infant alarm limits } & & & & $\mathrm{NS}^{\mathrm{a}, \mathrm{b}}$ \\
\hline & Yes, $n / N(\%)$ & $23 / 29(79 \%)$ & $15 / 22(68 \%)$ & \\
\hline & No, $n / N(\%)$ & $6 / 29(21 \%)$ & $7 / 22(32 \%)$ & \\
\hline \multicolumn{5}{|l|}{ Term saturation targets } \\
\hline & $\begin{array}{l}\text { Lower, median } \\
\text { (min, max) }\end{array}$ & $91(85,96),(n=35)$ & $91(70,96),(n=19)$ & $\mathrm{NS}^{\mathrm{a}, \mathrm{c}}$ \\
\hline & $\begin{array}{l}\text { Upper, median } \\
\text { (min, max) }\end{array}$ & $96(90,100),(n=35)$ & $100(89,100),(n=19)$ & $\mathrm{NS}^{\mathrm{a}, \mathrm{c}}$ \\
\hline \multicolumn{5}{|l|}{ Term alarm limits } \\
\hline & $\begin{array}{l}\text { Lower, median } \\
\text { (min, max) }\end{array}$ & $89(69,94),(n=23)$ & $89(79,95),(n=15)$ & $\mathrm{NS}^{\mathrm{a}, \mathrm{c}}$ \\
\hline & $\begin{array}{l}\text { Upper, median } \\
(\min , \max )\end{array}$ & $97(91,100),(n=23)$ & $100(96,100),(n=15)$ & $\mathrm{NS}^{\mathrm{a}, \mathrm{c}}$ \\
\hline
\end{tabular}

${ }^{a}$ Not significant, ( $p$ value $>0.0009$ ), based on Bonferroni Correction.

${ }^{\mathrm{b}} p$ value calculated with Chi-squared $\left(\chi^{2}\right)$ test.

${ }^{c} p$ value calculated with Wilcoxon signed rank-sum test. neonatal nurses) in sub-Saharan Africa will improve access to oxygen/air blending and oxygen saturation monitoring to prevent an epidemic of blindness due to ROP in subSaharan Africa. Assuring access to equipment to blend oxygen/air and to monitor oxygen saturation, along with education and quality assurance on their use, are likely to reduce blindness from ROP in sub-Saharan Africa. We aim to address these challenges through distributing the results of this study to survey participants, by providing training and equipment to facilitate the proper management of oxygen supplementation in preterm infants in sub-Saharan Africa, and by collecting additional data on the incidence of severe ROP and impact of improved oxygen management in collaboration with providers in neonatal care units within sub-Saharan Africa via the SIBA project.

Acknowledgements Charlotte Tibi translated the survey into French and Ruth Baptista translated the survey into Portuguese. Liam Epp assisted with the statistical analysis of the data.

Author contributions SKH conceived and designed the study, performed the literature review, acquired the data, performed data analysis, and drafted the manuscript. AS, YEV, SRL, SJI, and VLY conceived and designed the study and acquired the data. VCE acquired the data. WAC conceived and designed the study and performed data analysis. All authors contributed to the manuscript revision and approved the final manuscript as submitted and agree to be accountable for all aspects of the work. 
Funding SKH received time support for this project from Brigham Young University College of Life Sciences. The other authors received no external funding.

\section{Compliance with ethical standards}

Conflict of interest SKH declares time support during research project from Brigham Young University College of Life Sciences. WAC declares personal fees from Mednax, outside the submitted work. The other authors have no conflict of interests to disclose.

Publisher's note Springer Nature remains neutral with regard to jurisdictional claims in published maps and institutional affiliations.

\section{References}

1. Darlow BA, Husain S. Primary prevention of ROP and the oxygen saturation targeting trials. Semin Perinatol. 2019;43:333-40.

2. Gilbert C, Rahi J, Eckstein M, O'Sullivan J, Foster A. Retinopathy of prematurity in middle-income countries. Lancet. 1997;350:12-4.

3. Gilbert C. Retinopathy of prematurity: A global perspective of the epidemics, population of babies at risk and implications for control. Early Hum Dev. 2008;84:77-82.

4. Shah PK, Narendran V, Kalpana N. Aggressive posterior retinopathy of prematurity in large preterm babies in South India. Arch Dis Child Fetal Neonatal Ed. 2012;97:F371-5.

5. Askie LM, Henderson-Smart DJ, Ko H. Restricted versus liberal oxygen exposure for preventing morbidity and mortality in preterm or low birth weight infants. Cochrane Database Syst Rev. 2009, https://doi.org/10.1002/14651858.CD001077.pub2/abstract.

6. Askie LM, Darlow BA, Finer N, Schmidt B, Stenson B, TarnowMordi W, et al. Association between oxygen saturation targeting and death or disability in extremely preterm infants in the neonatal oxygenation prospective meta-analysis collaboration. JAMA. 2018;319:2190-201.

7. Gantz MG, Carlo WA, Finer NN, Rich W, Faix RG, Yoder BA, et al. Achieved oxygen saturations and retinopathy of prematurity in extreme preterms. Arch Dis Child Fetal Neonatal Ed. 2020;105:138-44.

8. Sink DW, Hope SAE, Hagadorn JI. Nurse:patient ratio and achievement of oxygen saturation goals in premature infants. Arch Dis Child Fetal Neonatal Ed. 2011;96:F93-8.

9. Gordillo L, Villanueva AM, Quinn GE. A practical method for reducing blindness due to retinopathy of prematurity in a developing country. J Perinat Med. 2012;40:577-82.

10. Gilbert C, Malik ANJ, Nahar N, Das SK, Visser L, Sitati S, et al. Epidemiology of ROP update - Africa is the new frontier. Semin Perinatol. 2019;43:317-22.

11. Gathara D, Serem G, Murphy GAV, Obengo A, Tallam E, Jackson D, et al. Missed nursing care in newborn units: a cross-sectional direct observational study. BMJ Qual Saf. 2020;29:19-30.

12. Cavicchiolo ME, Lanzoni P, Wingi MO, Pizzol D, Daverio M, Da Dalt L, et al. Reduced neonatal mortality in a regional hospital in Mozambique linked to a quality improvement intervention. BMC Pregnancy Childbirth. 2016;16:366.

13. Lloyd T, Isenberg S, Lambert SR. Current management of retinopathy of prematurity in sub-Saharan Africa. $J$ of AAPOS. 2020;24:151.e1-151.e6.

14. Oxygen therapy for children: a manual for health workers. World Health Organization: Geneva, Switzerland, 2016.
15. Varughese S, Gilbert C, Pieper C, Cook C. Retinopathy of prematurity in South Africa: an assessment of needs, resources and requirements for screening programmes. $\mathrm{Br} \mathrm{J}$ Ophthalmol. 2008;92:879-82.

16. Orimadegun AE, Ogunbosi BO, Akinbami FO. Knowledge and views of paediatricians about pulse oximetry: a nationwide online survey in Nigeria. Afr J Respir Med. 2011;7:14-8.

17. Belle JM, Cohen HJ, Shindo N, Lim ML, Velazquez-Berumen A, Ndihokubwayo J-B, et al. Influenza preparedness in low-resource settings: a look at oxygen delivery in 12 African countries. J Infect Dev Ctries. 2010;4:419-24.

18. Melesse MA, Gilbert C, Kerie A. Retinopathy of prematurity - an emerging cause of childhood blindness in Ethiopia. Ethiop Med J. 2020;58:167-72.

19. Kabesha TB, Kabesha D, Rehema G, Mukanire B, Iye S, Luembe $\mathrm{D}$, et al. [Result of intravitreal injection of Bevacizumab (Avastin) in the treatment of retinopathy of prematurity (About 11 cases followed up at Bukavu from 01.012014 to 06.30 2016)]. Lubumbashi Med J Fac Med. 2017;1:1-12.

20. Saleem M, Abdalla N. Screening of retinopathy of prematurity in Khartoum City, Sudan, 2011-2012. Merit Res J Microbiol Biol Sci. 2014;2:25-30.

21. Gezmu AM, Shifa JZ, Quinn GE, Nkomazana O, Ngubula JC, Joel D, et al. Incidence of retinopathy of prematurity in Botswana: a prospective observational study. Clin Ophthalmol. 2020;14: 2417-25.

22. Mutangana F, Muhizi C, Mudereva G, Noë P, Musiime S, Ngambe T, et al. Retinopathy of prematurity in Rwanda: a prospective multi-centre study following introduction of screening and treatment services. Eye. 2020;34:847-56.

23. Omer IM, Hassan HA. The prevalence and risk factors of retinopathy of prematurity among preterm babies admitted to Soba Neonatal Intensive Care Unit. Sudan J Paediatr. 2014;14: $17-21$.

24. Sitati SM, Ojuma SM, de Alba CAG. Retinopathy of prematurity: prevalence and risk factors among infants in rural Kenya. J Ophthalmol East Cent South Afr. 2018;22:63-7.

25. Braimah IZ, Enweronu-Laryea C, Sackey AH, Kenu E, Agyabeng $\mathrm{K}$, Ofori-Adjei I-OD-B, et al. Incidence and risk factors of retinopathy of prematurity in Korle-Bu Teaching Hospital: a baseline prospective study. BMJ Open. 2020;10:e035341.

26. Coghill M, Ambalavanan N, Chatburn RL, Hibberd PL, Wright LL, Carlo WA. Accuracy of a novel system for oxygen delivery to small children. Pediatrics. 2011;128:e382-7.

27. Floersch J, Hauschildt E, Keester A, Poganski S, Tran K, Slusher $\mathrm{T}$, et al. A low-resource oxygen blender prototype for use in modified bubble CPAP circuits. J Med Devices. 2020;14. 0150011-10 https://asmedigitalcollection.asme.org/medicaldevices/a rticle/14/1/015001/1072428/A-Low-Resource-Oxygen-BlenderPrototype-for-Use-in.

28. Kana H, Mayet I, Soma D, Alli HD, Biddulph S. The efficacy of intravitreal antivascular endothelial growth factor as primary treatment of retinopathy of prematurity: experience from a tertiary hospital. S Afr Med J. 2017;107:215-8.

29. du Bruyn A, Visser L. Eight years of treatable retinopathy of prematurity in KwaZulu-Natal: are we winning the battle? South Afr Ophthalmol J. 2017;12:8-10.

30. VanderVeen DK, Melia M, Yang MB, Hutchinson AK, Wilson LB, Lambert SR. Anti-vascular endothelial growth factor therapy for primary treatment of type 1 retinopathy of prematurity: a report by the American Academy of Ophthalmology. Ophthalmology. 2017;124:619-33. 